\title{
Study Site Protocol Version Relationship Effective Date Range
}

National Cancer Institute

\section{Source}

National Cancer Institute. Study Site Protocol Version Relationship Effective Date Range. NCI Thesaurus. Code C94151.

The date and time span specifying when the relationship between a study site and protocol version begins and ends. 